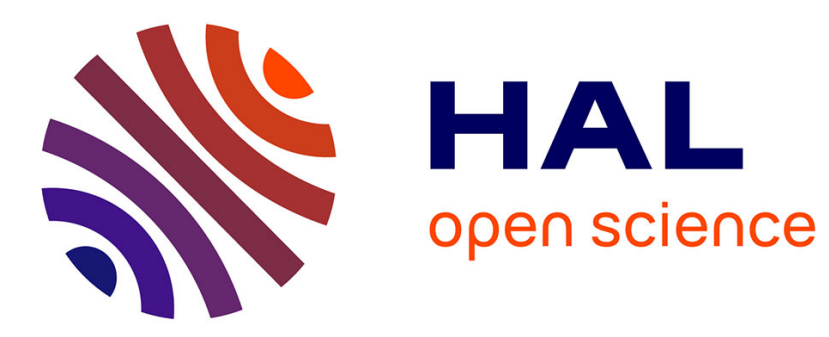

\title{
The Topological Gradient Method: From Optimal Design to Image Processing
}

\author{
Stanislas Larnier, Jérôme Fehrenbach, Mohamed Masmoudi
}

\section{To cite this version:}

Stanislas Larnier, Jérôme Fehrenbach, Mohamed Masmoudi. The Topological Gradient Method: From Optimal Design to Image Processing. Milan Journal of Mathematics, 2012, 80 (2), pp.411441. 10.1007/s00032-012-0196-5 . hal-01333752

\section{HAL Id: hal-01333752 https://hal.science/hal-01333752}

Submitted on 19 Jun 2016

HAL is a multi-disciplinary open access archive for the deposit and dissemination of scientific research documents, whether they are published or not. The documents may come from teaching and research institutions in France or abroad, or from public or private research centers.
L'archive ouverte pluridisciplinaire HAL, est destinée au dépôt et à la diffusion de documents scientifiques de niveau recherche, publiés ou non, émanant des établissements d'enseignement et de recherche français ou étrangers, des laboratoires publics ou privés. 


\title{
The topological gradient method: From optimal design to image processing
}

\author{
Stanislas Larnier, Jérôme Fehrenbach and Mohamed Masmoudi
}

\begin{abstract}
The aim of this article is to review and extend the applications of the topological gradient to major image processing problems. We briefly review the topological gradient, and then present its application to the crack localization problem, which can be solved using the Dirichlet to Neumann approach. A very natural application of this technique in image processing is the inpainting problem, which can be solved by identifying the optimal location of the missing edges. Edge detection is of extreme importance, as edges convey essential information in a picture. A second natural application is then the image reconstruction. A class of image reconstruction problems is considered that includes restoration, demosaicing, segmentation and super-resolution. These problems are studied using a unified theoretical framework which is based on the topological gradient method. This tool is able to find the localization and orientation of the edges for blurred, low sampled, partially masked, noisy images. We review existing algorithms and propose new ones. The performance of our approach is compared with conventional image reconstruction processes.
\end{abstract}

Mathematics Subject Classification (2010). 94A08, 49Q10, 49Q12.

Keywords. Edge detection, topological gradient, anisotropic diffusion, image denoising, demosaicing, image inpainting, image segmentation, super-resolution.

\section{Introduction}

Topology optimization formulates a design problem as an optimal material distribution problem. The search of an optimal domain is equivalent to finding its characteristic function, it is a 0-1 optimization problem. It can be seen as a generalization of shape optimization because it does not impose restriction on the topology of the optimal shape, for example the number of connected components of the domain or of its complementary. 
Different approaches make this problem differentiable:

$>$ Relaxation, homogenization,

$>$ Level set,

$>$ Topological gradient.

The homogenization technique [3, 2, 22, 21] is based on relaxed formulations leading to the introduction of some intermediate material or microstructures. The drawback is precisely that the optimal solution is not a classical design: it is a distribution of composite materials. Then penalization methods must be applied in order to retrieve a feasible shape. This method has mainly been applied to linear elasticity and particular objective functions.

The level set method [51, 50, 5, 60, can handle boundary propagation with topological changes. In practice, the level set method can easily remove holes but cannot create new ones. For this reason, in [4, the authors couple the level set method with the topological gradient method, which allow the creation of new holes in the optimization process.

This work focuses on the topological gradient [35, 57, 58, 46, 35, 38, 39, 7, 8, 47. To present the basic idea, let $\Omega$ be a domain of $\mathbb{R}^{d}, d \in \mathbb{N} \backslash\{0\}$ and $j(\Omega)=J\left(u_{\Omega}\right)$ a cost function to be minimized, where $u_{\Omega}$ is a solution to a given partial differential equation defined in $\Omega$. Let $x$ be a point in $\Omega$ and $\omega_{1}$ a smooth open bounded subset in $\mathbb{R}^{d}$ containing the origin. For a small parameter $\rho>0$, let $\Omega \backslash \bar{\omega}_{\rho}$ be the perturbed domain obtained by making a perforation $\omega_{\rho}=\rho \omega_{1}$ around the point $x$. The topological asymptotic expansion of $j\left(\Omega \backslash \overline{\omega_{\rho}}\right)$ when $\rho$ tends to zero is the following:

$$
j\left(\Omega \backslash \overline{\omega_{\rho}}\right)=j(\Omega)+f(\rho) g(x)+o(f(\rho)) .
$$

In this expansion, $f(\rho)$ denotes an explicit positive function going to zero with $\rho$ and $g(x)$ is called the topological gradient or topological derivative. It is usually simple to compute and is obtained using the solution of direct and adjoint problems defined on the initial domain. To minimize the criterion $j$, one has to create holes at some points where the topological gradient is negative.

The topological gradient has been applied to different kinds of topology optimization problems:

$>$ the elasticity case 35,

$>$ the Poisson equation [38,

$>$ the Navier-Stokes equation [6],

$>$ the Helmholtz equation [8,

$>$ the heat equation 9 ]

$>$ the wave equation 9 ]

In this work, the topological gradient method is applied to image processing problems. Topology optimization and image processing share a common goal: the partitioning of a given domain. In topology optimization, the goal is to look for the optimal design and its complementary. In image processing, a very common problem is to split the image in an edge set and its 
complementary. For this reason, topology optimization and image processing problems share common mathematical methods like variational methods, level set approaches, and topological gradient.

In our context, $\Omega$ is an open bounded domain of $\mathbb{R}^{2}$ and edges are modeled by cracks. We have just to adapt the topological asymptotic expansion, presented above 1.1. to the particular case of the crack. A crack $\sigma(x, \mathbf{n}, \rho)$ is a straight line of length $2 \rho$, centered at the point $x$ of $\Omega$, and normal to the unit vector $\mathbf{n}$. For a small $\rho \geq 0$, let $\Omega_{\rho}=\Omega \backslash \sigma(x, \mathbf{n}, \rho)$ be the perturbed domain by the insertion of a small crack.

The topological sensitivity theory provides a topological asymptotic expansion of $j$ when $\rho$ tends to zero. It takes the general form

$$
j\left(\Omega_{\rho}\right)-j(\Omega)=f(\rho) g(x)+\circ(f(\rho)) .
$$

To improve the minimization process, the topological gradient is defined by

$$
g(x)=\min _{n} g(x, n) .
$$

Using this gradient type information, it is possible to build fast algorithms. In most applications, a satisfying approximation of the optimal solution is reached at the first iteration of the optimization process.

We will consider the inverse conductivity problem, also known as the Calderon problem [26], consists in identifying the coefficients of a diffusion operator from the knowledge of the Dirichlet to Neumann operator. This problem has been widely studied in literature [33, 34, 42, 43]. In the particular case of cracks identification, the problem seems to be more convenient to solve thanks to the singularities of the solution. Only two measurements are needed to recover several simple cracks [1, 18, 7]. From the numerical point of view, several methods $[10,19,20,23,24,33,49,56$, have been proposed, but the topological gradient approach seems to be the most efficient method for crack localization [7].

The basic idea of the application of topological gradient to image processing is to adapt this crack localization method: an image can be viewed as a piecewise smooth function and edges can be modeled by a set of singularities or cracks [17, 14]. The idea of relaxing continuity constraints through the choice of a non-constant diffusion coefficient is very natural [59, but the topological gradient approach provides an accurate identification of the discontinuities.

As a straightforward application of the Calderon problem to image processing, we consider the inpainting problem. The goal of inpainting is to fill a hidden part of an image. In other words, if we denote by $\Omega$ the domain of the original image and $\omega$ the hidden part of the image, our goal is to recover the hidden part from the known part of the image in $\Omega \backslash \omega$. Here the interior of $\omega$ is not empty: it is not a sparse random set nor a narrow line. 
This problem has been widely studied and many methods have been considered:

> Learning approaches (neural networks, radial basis functions, support vector machine, ... ); the learning data is taken in $\Omega \backslash \omega$, then the approximate model is evaluated in $\omega$ [65, 66],

$>$ Minimization of an energy cost function in $\omega$ based on a total variation norm [28, 29],

$>$ Morphological component analysis methods separating texture and cartoon 32 .

Crack detection allows to identify the edges of the hidden part of the image, and the inpainting problem is then solved easily.

As a second application of this crack identification technique, we consider a general image reconstruction problem. This paper addresses linear inverse problems defined as follows. Let $u$ be an original image, $v$ the observed image, $L$ a linear observation operator and $n$ an additive noise. These quantities are related by

$$
v=L u+n \text {. }
$$

The objective is to reconstruct $u$ from $v$. A typical example is the superresolution image reconstruction, the objective being to recover a high-definition image from one or a number of noisy filtered and sub-sampled images [52, 37]. In this case, the observation operator $L$ is the composition of a filter and a sub-sampling operator.

The first contribution of this paper is to present a general framework based on the specification of the observation operator $L$. This framework is then applied to different image processing problems: image restoration, inpainting, demosaicing, segmentation and super-resolution. In these applications, edge detection is crucial, as edges convey essential information in a picture.

The topological gradient has been used in restoration, classification, inpainting and segmentation [17, 14, 15, 16, 44]. This technique can be applied to gray-level and color images, but also to three-dimensional images, or movies.

In the majority of previous works, only a part of the information derived from the topological asympotic expansion is used. The edges are detected using topological gradient and in a second step an isotropic diffusion is applied with two coefficients: a small coefficient on the edges, and a large coefficient on smooth parts. The second contribution of the present work is to propose a reconstruction algorithm (Algorithm 2) that takes into account the complete information provided by the topological asymptotic analysis: the edges are detected, and their orientation is also used. This additional information is used to define an anisotropic diffusion tensor on the edges. Outside the edges the diffusion tensor is isotropic, and the diffusion coefficient depends on the value of the topological gradient. 
The general framework and the reconstruction algorithm mentioned above are illustrated by a variety of examples: restoration, segmentation, inpainting, demosaicing and super-resolution. These examples are compared to the existing isotropic reconstruction algorithm, and to conventional methods.

Section 2 recalls our method for crack localization using the topological gradient. Section 3 presents the adaptation of this technique to inpainting. Section 4 is dedicated to the presentation and theoretical study of the general framework. In Section 5, the image reconstruction algorithm (Algorithm 2) and the numerical implementation are presented. Section 6 considers the case of the restoration algorithm [17, 15] where $L$ is the identity map and proposes an improvement of existing algorithms taking into account anisotropic diffusion. Texture removal is also presented in this section. Demosaicing and inpainting problems when $L$ is only a sub-sampling operator are treated in Section 7. Finally, Section 8 is dedicated to super-resolution image reconstruction.

\section{Crack localization problem}

\subsection{Dirichlet and Neumann problems}

We recall in this section the crack detection technique presented in [7]. Let $\Omega$ be a bounded open set of $\mathbb{R}^{2}$. We assume that there is a perfectly insulating crack $\sigma^{*}$ inside the domain. We impose a flux $\phi \in H^{-1 / 2}(\partial \Omega)$ on the boundary $\partial \Omega$ of $\Omega$, and for a given crack $\sigma \subset \Omega$, we consider the solution $u \in H^{1}(\Omega \backslash \sigma)$ to

$$
\begin{cases}\Delta u=0 & \text { in } \Omega \backslash \sigma \\ \partial_{n} u=\phi & \text { on } \partial \Omega \\ \partial_{n} u=0 & \text { on } \sigma .\end{cases}
$$

In order to have a well-posed direct problem 2.1), we assume that

$$
\int_{\partial \Omega} \phi d s=0
$$

and

$$
\int_{\Omega \backslash \sigma} u d x=0 .
$$

Moreover, the solution $u$ to 2.1 has to satisfy $u_{\mid \partial \Omega}=T$ where $T$ is a given function of $H^{1 / 2}(\partial \Omega)$. This additional boundary condition could be satisfied by finding optimal distributions of cracks inside the domain $\Omega$.

As we have an over-determination in the boundary conditions, we can define a Dirichlet and a Neumann problem:

$$
\text { Find } u_{D} \in H^{1}(\Omega \backslash \sigma) \text { such that }\left\{\begin{array}{l}
\Delta u_{D}=0 \text { in } \Omega \backslash \sigma, \\
u_{D}=T \text { on } \partial \Omega, \\
\partial_{n} u_{D}=0 \text { on } \sigma,
\end{array}\right.
$$


and

$$
\text { find } u_{N} \in H^{1}(\Omega \backslash \sigma) \text { such that } \begin{cases}\Delta u_{N}=0 & \text { in } \Omega \backslash \sigma, \\ \partial_{n} u_{N}=\phi & \text { on } \partial \Omega, \\ \partial_{n} u_{N}=0 & \text { on } \sigma \text {. }\end{cases}
$$

The normal derivative of the solution is equal to zero on both sides of $\sigma$. The gradient of the solution is tangent to the crack if we consider the domain $\Omega \backslash \sigma$, but in the domain $\Omega$ the gradient is defined in the distribution sense and is normal to the crack. We are on the edge of the image.

It is clear that for the actual crack $\sigma^{*}$, the two solutions $u_{D}$ and $u_{N}$ are equal. The idea is then to consider the following cost function:

$$
J(\sigma)=\frac{1}{2}\left\|u_{D}-u_{N}\right\|_{L^{2}(\Omega)}^{2},
$$

where $u_{D}$ and $u_{N}$ are solutions to problems 2.4 and 2.5 respectively for the given crack $\sigma$.

\subsection{Minimization by topological asymptotic analysis}

We consider in this section the two corresponding adjoint states, respectively solutions in $H^{1}(\Omega)$ to

$$
\left\{\begin{array}{l}
-\Delta p_{D}=-\left(u_{D}-u_{N}\right) \text { in } \Omega, \\
p_{D}=0 \text { on } \partial \Omega
\end{array}\right.
$$

and

$$
\left\{\begin{array}{l}
-\Delta p_{N}=+\left(u_{D}-u_{N}\right) \quad \text { in } \Omega \\
\partial_{n} p_{N}=0 \text { on } \partial \Omega .
\end{array}\right.
$$

The variation of the cost function $j(\rho):=J\left(u_{\sigma(x, \mathbf{n}, \rho)}\right)$ induced by the insertion of this small crack is given by the topological gradient theory [7]:

$$
j(\rho)-j(0)=f(\rho) g(x, \mathbf{n})+o(f(\rho)),
$$

where $f(\rho)=\pi \rho^{2}$ and $g$ is given by

$$
g(x, \mathbf{n})=-\left[\left(\nabla u_{D}(x) \cdot \mathbf{n}\right)\left(\nabla p_{D}(x) \cdot \mathbf{n}\right)+\left(\nabla u_{N}(x) \cdot \mathbf{n}\right)\left(\nabla p_{N}(x) \cdot \mathbf{n}\right)\right] .
$$

The solutions $u_{D}, u_{N}, p_{D}$, and $p_{N}$ are calculated in the initial domain $\Omega$ without any crack. The topological gradient can then be rewritten in the following way

$$
g(x, \mathbf{n})=\mathbf{n}^{T} M(x) \mathbf{n},
$$

where $M(x)$ is the $2 \times 2$ symmetric matrix defined by

$$
M(x)=-\operatorname{sym}\left(\nabla u_{D}(x) \cdot \nabla p_{D}(x)^{T}+\nabla u_{N}(x) \cdot \nabla p_{N}(x)^{T}\right) .
$$

We can deduce that $g(x, \mathbf{n})$ is minimal when the normal $\mathbf{n}$ is the eigenvector associated to the smallest (i.e. most negative) eigenvalue of the matrix $M(x)$. In the following, this eigenvalue will be considered as the topological gradient.

We can then define a simple and very fast numerical algorithm. First, we solve the two direct problems (Dirichlet and Neumann), and the two corresponding adjoint problems. Then, at each point $x$ of the domain, we 

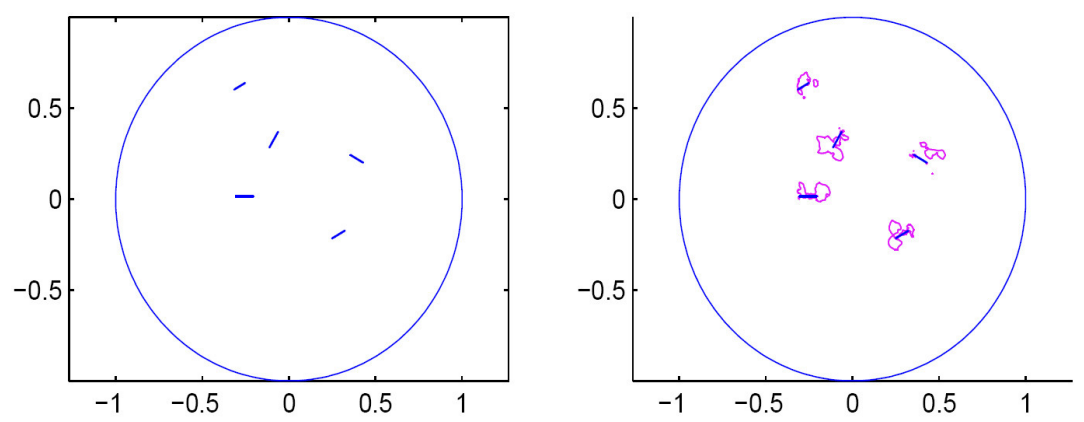

FIGURE 1. Left: actual cracks; right: superposition of the actual cracks and a topological gradient isovalue. Figure extracted from [7.

compute the matrix $M(x)$ and its two eigenvalues. The crack likely lies in the most negative gradient regions.

Applying the procedure described above, an example of localization of the unknown cracks using the topological gradient is shown in Figure 1 (extracted from [7). The most negative values of the topological gradient are located around the actual cracks, and these results are obtained in only one iteration.

\section{Application to inpainting problems}

Our image inpainting approach is based on the hypothesis that the image is regular outside edges. We suppose even that it is harmonic $(\Delta u=0)$. Of course, this assumption is not valid in real applications, but it gives a theoretical justification of our algorithm, which still works if the image is not harmonic outside edges. The basic idea is then to create a harmonic extension of the image from boundary estimation of $u$ and $\partial_{n} u$. This analytic extension is unique when it exists. The cracks identification process gives the boundary of the domain of validity of the extension.

\subsection{Algorithm}

We denote by $\Omega$ the image and $\partial \Omega$ its boundary, $\omega$ the missing part of the image and $\gamma$ its boundary. In the following $v$ represents the image we want to restore, $T$ will represent here the value of the image on the boundary of the missing zone, and $\phi$ will be the corresponding flux. We have then $T=v$ and $\phi=\partial_{n} v$ in the corresponding domains. Theoretically, we have to assume $v$ to be enough regular, for example in $H^{2}(\Omega)$, but it will be possible to work with $v$ in $L^{2}(\Omega)$. 
We now consider the problem of finding $u_{\alpha} \in H^{1}(\Omega)$, solution to the following equation:

$$
\left\{\begin{array}{l}
-\alpha \Delta u_{\alpha}+u_{\alpha} \cdot \chi_{\Omega \backslash \omega}=v \cdot \chi_{\Omega \backslash \omega} \quad \text { in } \Omega, \\
\partial_{n} u_{\alpha}=0 \text { on } \partial \Omega
\end{array}\right.
$$

where $\alpha$ is a small positive number. This equation can be rewritten as

$$
\text { find } u_{\alpha} \in H^{1}(\Omega) \text { such that }\left\{\begin{array}{l}
-\alpha \Delta u_{\alpha}+u_{\alpha}=v \text { in } \Omega \backslash \omega, \\
\Delta u_{\alpha}=0 \text { in } \omega \\
\partial_{n} u_{\alpha}=0 \text { on } \partial \Omega
\end{array}\right.
$$

When $\alpha \rightarrow 0$, it is in some sense equivalent when $v$ is regular to find the solution $u_{N} \in H^{1}(\Omega \backslash \gamma)$ to

$$
\left\{\begin{array}{l}
u_{N}=v \quad \text { in } \Omega \backslash \omega \\
\Delta u_{N}=0 \quad \text { in } \omega \\
\partial_{n} u_{N}=\partial_{n} v \text { on } \gamma
\end{array}\right.
$$

which can be seen as a Neumann problem in $\omega$.

From a numerical point of view, we will solve equation 3.2 with a very small positive $\alpha$ and we will consider that it is our Neumann problem. The Dirichlet problem does not require any special care.

The inpainting algorithm is then the following:

\section{Inpainting algorithm:}

- Calculation of $u_{D}$ and $u_{N}$, respectively solutions to

$$
\left\{\begin{array}{l}
u_{D}=v \quad \text { in } \Omega \backslash \omega \\
\Delta u_{D}=0 \text { in } \omega \\
u_{D}=v \text { on } \gamma
\end{array}\right.
$$

where $u_{D} \in H^{1}(\Omega)$, and

$$
\left\{\begin{array}{l}
-\alpha \Delta u_{N}+u_{N}=v \quad \text { in } \Omega \backslash \omega \\
\Delta u_{N}=0 \text { in } \omega \\
\partial_{n} u_{N}=0 \text { on } \partial \Omega
\end{array}\right.
$$

where $u_{N} \in H^{1}(\Omega)$ (the normal derivative is the same on the two sides of $\gamma$ ).

- Calculation of $p_{D}$ and $p_{N}$ the two corresponding adjoint states, respectively solutions to

$$
\left\{\begin{array}{l}
p_{D}=0 \quad \text { in } \Omega \backslash \omega \\
-\Delta p_{D}=-\left(u_{D}-u_{N}\right) \quad \text { in } \omega \\
p_{D}=0 \text { on } \gamma
\end{array}\right.
$$


and

$$
\left\{\begin{array}{l}
p_{N}=0 \text { in } \Omega \backslash \omega \\
-\Delta p_{N}=+\left(u_{D}-u_{N}\right) \quad \text { in } \omega \\
\partial_{n} p_{N}=0 \text { on } \gamma
\end{array}\right.
$$

- Computation of the $2 \times 2$ matrix $M(x)$ (see eq. 2.12) ) and its lowest eigenvalue $\lambda_{\min }$ at each point of the missing domain $\omega$.

- Definition of cracks localization: $\left\{x \in \omega ; \lambda_{\min }(x)<\delta<0\right\}$, where $\delta$ is a negative threshold.

- Calculation of $u$ solution to the Neumann problem (2.5) taking into account the cracks location.

It is not so easy to take into account cracks, but from a numerical point of view, cracks are modeled by a small conductivity.

This algorithm has a complexity of $\mathcal{O}(N \cdot \log (N))$, where $N$ is the number of pixels. See [15] for more details.

\subsection{Numerical results}

We have applied our algorithm to the inpainting of a real color image.

Figure 2 shows respectively the occluded part by a black word (a), the identified missing edges (b) and the corresponding inpainted image by our algorithm (c). One can see that the reconstruction of the missing part of the image is very satisfactory, and all the occluded part has been treated at the same time. We also applied a TV inpainting algorithm (see e.g. 30 for details about this method) to the same image, and the result is shown in Figure 2 (d).

Figure 3 allows to compare more in details the original image (a) and the inpainted images using topological gradient (b) and total variation (c) algorithms. One can notice the remarkable sharp edges produced by our algorithm, as contrast to TV inpainting that slightly blurs edges.

There is no need to split the image into several parts, and the occluded part can have any geometry. It can even be a part of the image boundary, as our algorithm also works when the intersection of the inpainting domain and the boundary of the image is not empty. This comes from the fact that the crack localization algorithm can be very easily adapted to incomplete data on the boundary [14]. Moreover, the reconstruction is done in only one iteration of the topological gradient algorithm, which consists in 5 resolutions of a PDE (the two direct and two adjoint problems, and then one direct problem) in the domain $\Omega$ representing the image. Using a discrete cosine transform and a preconditioned conjugate gradient approach, it is possible to speed up these resolutions, and we obtain finally a $\mathcal{O}(N \cdot \log (N))$ complexity where $N$ is the number of pixels (see [15] for more details). 


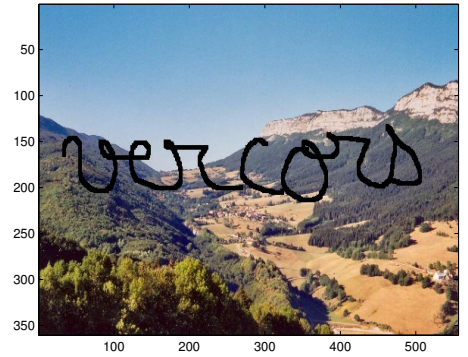

(a)

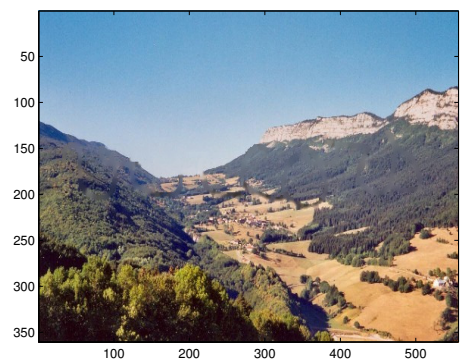

(c)

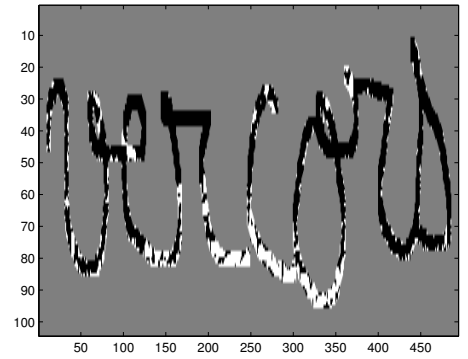

(b)

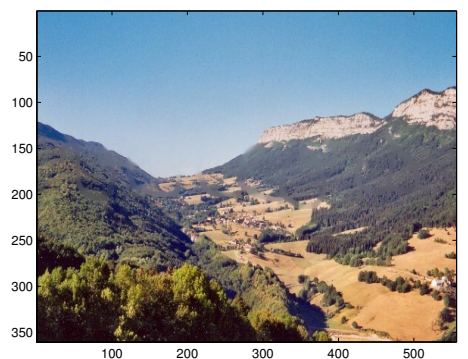

(d)

FIGURE 2. Inpainting of a color image: occluded image (a); identified missing edges by the topological gradient (b); corresponding inpainted images using our algorithm (c) and a TV inpainting algorithm (d). Figure extracted from [16].

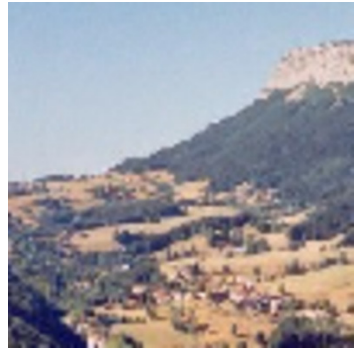

(a)

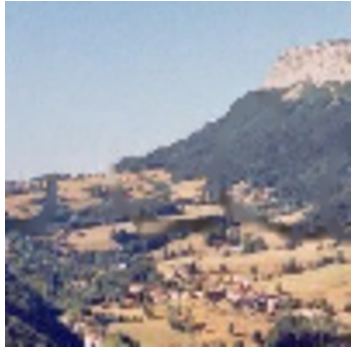

(b)

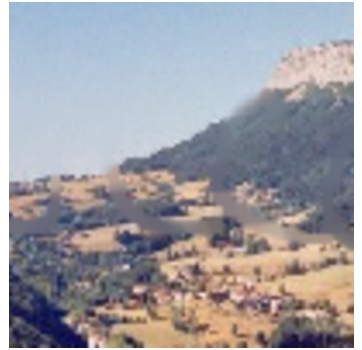

(c)

FigURE 3. Inpainting of a color image: zoom of original image (a); topological gradient inpainted image (b); TV inpainted image(c). Figure extracted from [16]. 


\section{General framework}

This work addresses the general problem of reconstructing an image $u \in$ $L^{2}(\Omega)$ given some noisy observations $L u+n$ in a Hilbert space $E$, where $\Omega$ is the (rectangular) domain where the image $u$ is defined. The observation space $E$ depends on the specific application as well as the linear observation operator $L: L^{2}(\Omega) \longrightarrow E$. The norm on the space $E$ will be written $\|\cdot\|_{E}$. Table 1 summarizes the different applications presented in this work. These will be detailed in Sections 6 to 8 .

\begin{tabular}{|c|c|c|}
\hline Specific application & space $E$ & $L u$ \\
\hline Restoration & $L^{2}(\Omega)$ & $u$ \\
\hline Inpainting & $L^{2}(\Omega \backslash \omega)$ & $u_{\mid \Omega \backslash \omega}$ \\
\hline Super-resolution with one image & $\mathbb{R}^{m \times n}$ & $\left((\varphi * u)\left(x_{i}\right)\right)_{1 \leq i \leq m n}$ \\
\hline Super-resolution with $k$ images & $\left(\mathbb{R}^{m \times n}\right)^{k}$ & $\left((\varphi * u)\left(x_{i}^{j}\right)\right)_{1 \leq i \leq m n, 1 \leq j \leq k}$ \\
\hline
\end{tabular}

TABLE 1. Applications of the general problem (2.1). The subset $\omega \subset \Omega$ is the region where the image has to be recovered, $\varphi$ is a filter (e.g. a Gaussian filter) and $x_{i} \in \Omega$, $i=1, \ldots, m n$ or $x_{i}^{j} \in \Omega, i=1, \ldots, m n, j=1, \ldots, k$ are the sampling points.

The general image reconstruction problem can be summed up by:

$$
\left\{\begin{array}{l}
\text { given } v=L u+n, \\
\text { reconstruct } u .
\end{array}\right.
$$

\subsection{The reconstruction problem}

The first idea to recover the original image is to minimize the following functional for $u \in H^{1}(\Omega)$ :

$$
\left\|c^{1 / 2} \nabla u\right\|_{L^{2}(\Omega)}^{2}+\|L u-v\|_{E}^{2},
$$

where $c$ is a positive constant or a positive definite tensor. The first term in Equation 4.2 ensures that the recovered image $u$ is regular, and the second term measures the discrepancy with the data. The reconstruction is improved by considering a diffusion coefficient $c(x)$ that depends on the space variable. In order to improve this method, nonlinear isotropic and anisotropic methods were introduced, we can cite here the work of Perona and Malik [53], Catté et al. 27] and more recently Weickert [62, 63] and Aubert [11]. Partial differential equations is not the only way to solve the restoration problem, as a representative to statistical methods, we can cite [31.

The present paper proposes an alternative construction of the spacedependent coefficient $c(x)$ based on topological asymptotic analysis. This construction is detailed in Section 5.1. We will assume in all this work that the following hypothesis is satisfied: 
Hypothesis 4.1. $\quad$ 1. $L$ is a linear bounded operator on $E$.

2. If $u=k$ in $\Omega$ with $k \in \mathbb{R}$ then $L u=k$.

In other words constant images in $\Omega$ are mapped to constants in $E$, with the same value. The adjoint operator $L^{*}$ of $L$ is also bounded.

The minimization of Equation 4.2 is equivalent to find $u \in H^{1}(\Omega)$ such that

$$
\left\{\begin{aligned}
-\nabla \cdot(c \nabla u)+L^{*} L u & =L^{*} v & & \text { in } \Omega, \\
\partial_{n} u & =0 & & \text { on } \partial \Omega,
\end{aligned}\right.
$$

where $\partial_{n}$ denotes the normal derivative to $\partial \Omega$.

The corresponding variational formulation can be written as:

$$
\left\{\begin{array}{l}
\text { find } u \in H^{1}(\Omega) \text { such that } \\
a_{0}(u, w)=\ell_{0}(w) \forall w \in H^{1}(\Omega),
\end{array}\right.
$$

where $a_{0}$ is the bilinear form, defined on $H^{1}(\Omega) \times H^{1}(\Omega)$ by

$$
a_{0}(u, w)=\int_{\Omega} c \nabla u \nabla w d x+\int_{\Omega} L u L w d x
$$

and $\ell_{0}$ is the linear form defined on $L^{2}(\Omega)$ by

$$
\ell_{0}(w)=\int_{\Omega} L^{*} v w d x .
$$

Lemma 4.2. Assume $c$ is a positive constant or a positive definite tensor. For $u \in H^{1}(\Omega)$, define $\|u\|_{a}=\sqrt{a_{0}(u, u)}$. Under Hypothesis 4.1. $\|\cdot\|_{a}$ is a norm equivalent to the norm $\|\cdot\|_{H^{1}(\Omega)}$

Lemma 4.2 follows directly from the general Poincar inequality and Hypothesis 4.1 .

Theorem 4.3. Under Hypothesis 4.1, the variational problem (4.4) has a unique solution.

Proof. To simplify the proof, the case $c=1$ is considered.

The first assertion of Hypothesis 4.1 implies that $a_{0}$ is a continuous bilinear form. Lemma 4.2 proves that $a_{0}$ is coercive. The following inequality

$$
\forall w \in L^{2}(\Omega) \quad\left|\ell_{0}(w)\right| \leq\left\|L^{*}\right\|\|v\|_{E}\|w\|_{L^{2}(\Omega)}
$$

proves that $\ell_{0}$ is a continuous linear form. The result follows from the LaxMilgram theorem.

\subsection{Perturbation of the domain and edge detection}

The solution $u$ of the problem 4.2 , or equivalently 4.3 is an element of $H^{1}(\Omega)$. This is a limitation of the method since it does not allow discontinuities in the recovered image. To overcome this limitation, discontinuities are included in the domain in the form of insulating cracks. This generalizes the concept introduced for the restoration equation in [17].

At a given point $x_{0} \in \Omega$, we insert a small insulating crack $\sigma_{\rho}=x_{0}+$ $2 \rho \sigma(\mathbf{n})$ where $\sigma(\mathbf{n})$ is a centred unit line segment, $\mathbf{n}$ is a unit vector normal to 


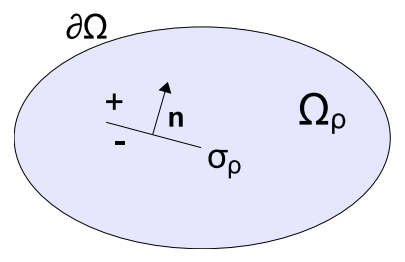

Figure 4. Cracked domain.

the crack and $2 \rho$ is the length of the crack. Let $\Omega_{\rho}=\Omega \backslash \sigma_{\rho}$ be the perturbed domain created by inserting this crack. The perturbed solution $u_{\rho} \in H^{1}\left(\Omega_{\rho}\right)$ satisfies

$$
\left\{\begin{aligned}
-\nabla \cdot\left(c \nabla u_{\rho}\right)+L^{*} L u_{\rho} & =L^{*} v & & \text { in } \Omega_{\rho}, \\
\partial_{n} u_{\rho} & =0 & & \text { on } \partial \Omega, \\
\partial_{n} u_{\rho} & =0 & & \text { on } \sigma_{\rho} .
\end{aligned}\right.
$$

The corresponding variational formulation is given by

$$
\left\{\begin{array}{l}
\text { find } u_{\rho} \in H^{1}\left(\Omega_{\rho}\right) \text { such that } \\
a_{\rho}\left(u_{\rho}, w\right)=\ell_{\rho}(w) \forall w \in H^{1}\left(\Omega_{\rho}\right),
\end{array}\right.
$$

where $a_{\rho}$ is the bilinear form, defined on $H^{1}\left(\Omega_{\rho}\right) \times H^{1}\left(\Omega_{\rho}\right)$ by

$$
a_{\rho}(u, w)=\int_{\Omega_{\rho}} c \nabla u \nabla w d x+\int_{\Omega_{\rho}} L^{*} L u w d x,
$$

and $\ell_{\rho}$ is the linear form, defined on $L^{2}\left(\Omega_{\rho}\right)$ by

$$
\ell_{\rho}(w)=\int_{\Omega_{\rho}} L^{*} v w d x
$$

When $\rho=0$ (i.e. the crack is reduced to a point) the solution of the variational formulation 4.6 coincides with the solution $u$ of the unperturbed problem (4.4).

The edge detection method consists in looking for a crack $\sigma$ such that the energy $j(\rho)=J_{\rho}\left(u_{\rho}\right)=\frac{1}{2} \int_{\Omega_{\rho}}\left|\nabla u_{\rho}\right|^{2}$ is as small as possible [17]. This amounts to state that the energy outside the edges is as small as possible.

The efficiency of topology optimization comes from the fact that the asymptotic variation of $j(\rho)$ as $\rho \rightarrow 0$ can be computed rapidly for any location $x_{0}$ and orientation $\mathbf{n}$ of the crack. It requires only to solve the direct problem 4.4 and the following adjoint problem

$$
\left\{\begin{array}{l}
\text { find } p_{0} \in H^{1}(\Omega) \text { such that } \\
a_{0}\left(w, p_{0}\right)=-\partial_{u} J\left(u_{0}\right)(w) \forall w \in H^{1}(\Omega) .
\end{array}\right.
$$

The precise statement is detailed in the following

Theorem 4.4. When $\rho \rightarrow 0$ the cost function $j$ has the following asymptotic expansion

$$
j(\rho)-j(0)=\rho^{2} g\left(x_{0}, \mathbf{n}\right)+o\left(\rho^{2}\right),
$$


where the topological gradient $g$ is given by

$$
g\left(x_{0}, \mathbf{n}\right)=-\pi c\left(\nabla u_{0}\left(x_{0}\right) \cdot \mathbf{n}\right)\left(\nabla p_{0}\left(x_{0}\right) \cdot \mathbf{n}\right)-\pi\left|\nabla u_{0}\left(x_{0}\right) \cdot \mathbf{n}\right|^{2} .
$$

The function $u_{0}$ and $p_{0}$ are supposed locally regular around $x_{0}$.

Proof. The following asymptotic expansions are proved in [7, 17, 14, 8]:

i)

$$
J_{\rho}\left(u_{\rho}\right)-J_{\rho}\left(u_{0}\right)=\partial_{u} J\left(u_{\rho}\right)\left(u_{\rho}-u_{0}\right)+\rho^{2} \delta J_{1}+o\left(\rho^{2}\right),
$$

where

$$
\delta J_{1}=-\pi\left|\nabla u_{0}\left(x_{0}\right) \cdot \mathbf{n}\right|^{2}
$$

ii)

iii)

$$
J_{\rho}\left(u_{0}\right)-J_{0}\left(u_{0}\right)=o\left(\rho^{2}\right)
$$

$$
\left(a_{\rho}-a_{0}\right)\left(u_{0}, p_{\rho}\right)=\rho^{2} \delta a+o\left(\rho^{2}\right),
$$

where the adjoint state $p_{\rho}$ is defined by

$$
\left\{\begin{array}{l}
\text { find } p_{\rho} \in H^{1}\left(\Omega_{\rho}\right) \text { such that } \\
a_{\rho}\left(w, p_{\rho}\right)=-\partial_{u} J\left(u_{\rho}\right)(w) \forall w \in H^{1}\left(\Omega_{\rho}\right)
\end{array}\right.
$$

and

$$
\delta a=-\pi c\left(\nabla u_{0}\left(x_{0}\right) \cdot \mathbf{n}\right)\left(\nabla p_{0}\left(x_{0}\right) \cdot \mathbf{n}\right)
$$

iv)

$$
\left(\ell_{\rho}-\ell_{0}\right)\left(p_{\rho}\right)=o\left(\rho^{2}\right) .
$$

Using Equations 4.10, 4.12, 4.14 and 4.6) we have

$$
\begin{aligned}
j(\rho)-j(0) & =J_{\rho}\left(u_{\rho}\right)-J_{0}\left(u_{0}\right) \\
& =\left(J_{\rho}\left(u_{\rho}\right)-J_{\rho}\left(u_{0}\right)\right)+\left(J_{\rho}\left(u_{0}\right)-J_{0}\left(u_{0}\right)\right) \\
& =\partial_{u} J\left(u_{\rho}\right)\left(u_{\rho}-u_{0}\right)+\rho^{2} \delta J_{1}+o\left(\rho^{2}\right) \\
& =\left(a_{\rho}-a_{0}\right)\left(u_{0}, p_{\rho}\right)+a_{0}\left(u_{0}, p_{\rho}\right)-a_{\rho}\left(u_{\rho}, p_{\rho}\right)+\rho^{2} \delta J_{1}+o\left(\rho^{2}\right) \\
& =\left(a_{\rho}-a_{0}\right)\left(u_{0}, p_{\rho}\right)-\left(\ell_{\rho}-\ell_{0}\right)\left(p_{\rho}\right)+\rho^{2} \delta J_{1}+o\left(\rho^{2}\right) .
\end{aligned}
$$

Applying Equations 4.13 and 4.16 end the proof.

The direct state $u_{0}$ and the adjoint state $p_{0}$ are computed in the initial domain without cracks. The topological gradient can be written $g(x, \mathbf{n})=$ $\mathbf{n}^{T} M(x) \mathbf{n}$, where $M(x)$ is the $2 \times 2$ symmetric matrix defined by

$$
M(x)=-\pi c \frac{\nabla u_{0}(x) \nabla p_{0}(x)^{T}+\nabla p_{0}(x) \nabla u_{0}(x)^{T}}{2}-\pi \nabla u_{0}(x) \nabla u_{0}(x)^{T} \text {. }
$$

For a given $x, g(x, \mathbf{n})$ takes its minimal value when $\mathbf{n}$ is the eigenvector associated to the lowest eigenvalue $\lambda_{\min }(x)$ of $M(x)$. This value is by definition the topological gradient associated to the optimal orientation of the crack $\sigma_{\rho}$ at the location $x . M(x)$ is composed of two terms, see Equation 4.17). The second term takes into account the structure tensor, like other methods in anisotropic diffusion [62] and corrects it with information given 
by the adjoint state included in the first term. The solution of the adjoint state contains information of higher order and is less sensitive to noise.

The edges are located at points $x$ where $\lambda_{\min }(x)$ is the most negative and their orientation is given by the corresponding eigenvector.

\subsection{Example of edge detection: the case of a noisy image}

The potential of topological gradient to detect edges and their orientation is illustrated here using an ellipse representation of the tensor $M(x)$ 4.17). In this first example the observation space is $E=L^{2}(\Omega)$ and the observation operator is $L=I d$.

Figure 5 presents an image (Barbara) perturbed by an additive Gaussian noise, and the topological gradient value $\lambda_{\min }(x)$ defined as the smallest eigenvalue of the matrix $M(x)$ defined by Equation 4.17).
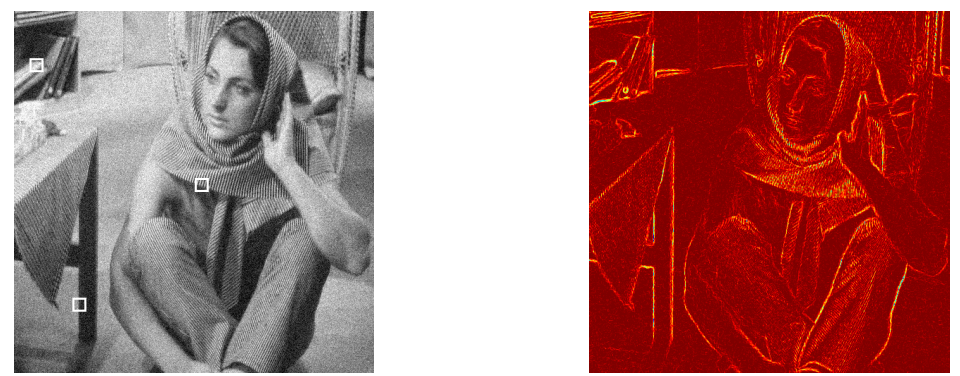

FIGURE 5. From left to right, noisy Barbara image and topological gradient.
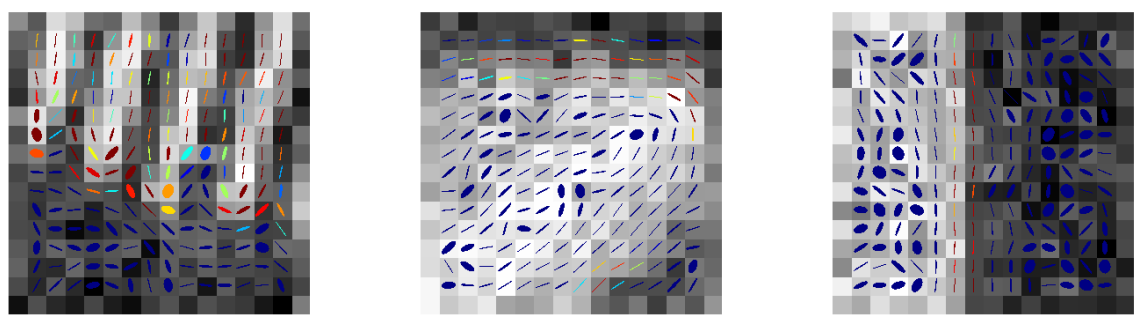

FiguRE 6. Ellipse representation of $M(x)$ : parts of noisy Barbara image 5. From left to right: scarf, books and table. The orientation of the axes of the ellipse is given by the eigenvectors of $M(x)$, the ratio of the semi-axes is the ratio of the eigenvalues, and the color of the ellipse depends on the value of $\lambda_{\min }(x)$ : the more negative is $\lambda_{\min }$, the redder is the ellipse. 
Figure 6 shows details of the image together with ellipses that represent, at each pixel of the image, the topological gradient tensor $M(x)$ 4.17). The orientation of the axes of the ellipse is given by the eigenvectors of $M(x)$, the ratio of the semi-axes corresponds to the ratio of the eigenvalues, and the color of the ellipse depends on the value of $\lambda_{\min }(x)$ : the more negative is $\lambda_{\min }$, the redder is the ellipse. The following observations confirm the efficiency of topological asymptotic analysis: the ellipses are oriented along the texture edges (the orientation is correct), and the homogeneous regions are in dark blue (the topological gradient is not very negative in homogeneous regions).

\section{Algorithms and numerical implementation}

\subsection{Algorithms}

In previous works on image processing using topological gradient [17, 15, 14, 16] the tensor $c$ is an isotropic tensor taking only two values: $c_{0}$ in the smooth part of the image and a small value $\epsilon$ on the edges. The edges are detected with a threshold $\delta$ on the topological gradient values. These ideas are summarized in Algorithm 11. which is the isotropic diffusion (ID) reconstruction algorithm. It is a generalization with an arbitrary observation operator $L$ of the algorithm presented in [17.

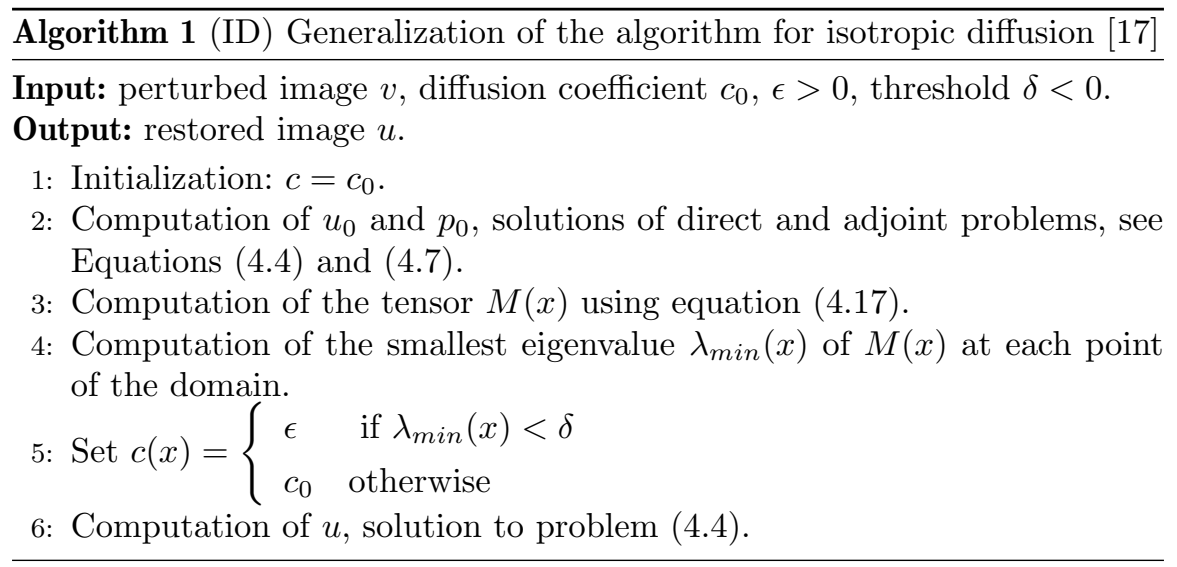

The present work proposes to take into account the whole information provided by topological asymptotic analysis, i. e. edge orientation and jump amplitude. These quantities allow to define a diffusion tensor $c(x)$ that is adapted to the image content.

More precisely the tensor $M(x)$ is computed using Equation (4.17). Let the smallest eigenvalue of $M(x)$ be denoted $\lambda_{\min }(x)$. The valley bottoms are local minima of $\lambda_{\text {min }}$ along the horizontal direction, the vertical direction or one of the diagonals. The edges are valley bottoms where $\lambda_{\min }$ is below a threshold $\delta$. The diffusion tensor $c(x)$ is anisotropic along the edges with the 
principal direction given by $M(x)$, and $c(x)$ is isotropic outside the edges. This is detailed in Algorithm 2 .

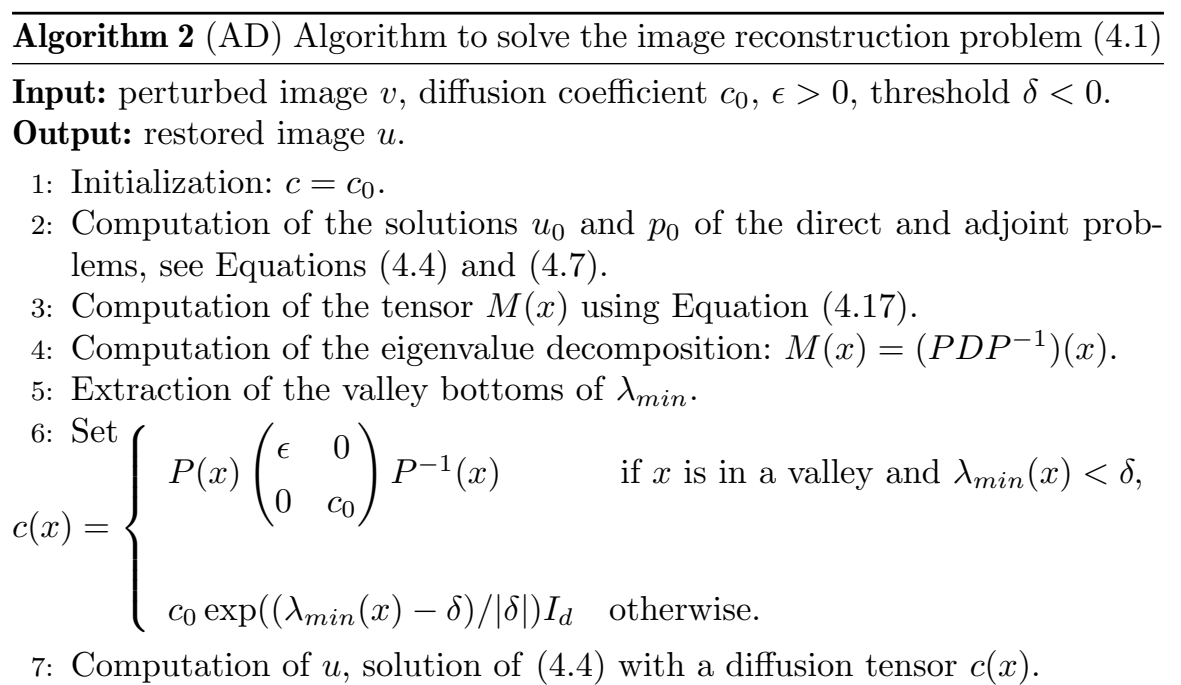

\subsection{Numerical implementation}

The algorithms were coded in MATLAB and equations 4.1) and (4.7) were solved with a finite difference method.

Our experiments used 8-bits images. The intensity of these images was divided by 255 , so we considered normalized images with an intensity in the interval $[0,1]$. When an additive Gaussian noise is addded, the standard deviation of the noise is denoted $\sigma$.

The different reconstructions are compared quantitatively using Peak Signal to Noise Ratio (PSNR) expressed in dB and the Structural SIMilarity (SSIM) 61. Let $I_{2}$ be a degraded image of an original image $I_{1}, m$ and $n$ be the image dimensions and ch the number of channels. The PSNR of $I_{2}$ is given by the following formula:

$$
\operatorname{PSNR}\left(I_{2}\right)=10 \log _{10}\left(\frac{m n c h}{\sum_{m, n}\left(I_{1}(i, j)-I_{2}(i, j)\right)^{2}}\right) .
$$

The SSIM of $I_{2}$ is given by the following formula:

$$
S S I M=\frac{\left(2 \mu_{1} \mu_{2}+c_{1}\right)\left(2 \sigma_{1,2}+c_{2}\right)}{\left(\mu_{1}^{2}+\mu_{2}^{2}+c_{1}\right)\left(\sigma_{1}^{2}+\sigma_{2}^{2}+c_{2}\right)},
$$

where $\mu_{1}$ is the average of $I_{1}, \mu_{2}$ is the average of $K, \sigma_{1}^{2}$ is the variance of $I_{1}$, $\sigma_{2}^{2}$ is the variance of $I_{2}, \sigma_{1,2}^{2}$ is the covariance of $I_{1}$ and $I_{2}, c_{1}=\left(k_{1} L\right)^{2}$ and $c_{2}=\left(k_{2} L\right)^{2}$ two variables to stabilize the division by a weak denominator (where $L$ is the dynamic range, usually $2^{\# \text { bits per pixel }}-1$, and $k_{1}=0.01$, $k_{2}=0.03$ by default) 
In order to perform a comparative evaluation, benchmark methods had to be chosen among many reconstruction methods for each application considered (restoration, demosaicing, super-resolution). Our choice was driven by two criteria :

$>$ the use of well-established methods,

$>$ the availability of efficient codes.

In these methods, some parameters often have to be adjusted. These parameters were chosen to optimize the results.

The color images were treated with a vectorial minimization problem, involving the resolution of vectorial problems. The topological asymptotic expansion is still given by Equation (4.8), 4.9 and (4.17), where all functions are vectorial, i.e. the topological gradient is the sum of the corresponding expressions for each channel 13 .

\section{The observation operator is $L=I d$}

A classical way to restore an image $u$ from its noisy version $v \in L^{2}(\Omega)$ is to find $u \in H^{1}(\Omega)$ which minimizes the following functional:

$$
\left\|c^{1 / 2} \nabla u\right\|_{L^{2}(\Omega)}^{2}+\|u-v\|_{L^{2}(\Omega)}^{2} .
$$

It is equivalent to solve the following PDE:

$$
\left\{\begin{aligned}
-\nabla \cdot(c \nabla u)+u & =v \text { in } \Omega, \\
\partial_{n} u & =0 \text { on } \partial \Omega,
\end{aligned}\right.
$$

where $c$ is a positive constant or a tensor and $\partial_{n}$ denotes the normal derivative to $\partial \Omega$. It is a particular case of Equation 4.3 with the observation operator $L=I d$. This equation and improvements using anisotropic diffusion have been the subject of numerous works [53, 54, 62, 11].

In Section 6.1, our Algorithm 2 is compared to the existing Algorithm 1 and to the Non-Local Means method [25] on one example. Section 6.2 is dedicated to results obtained when the same equation is used to remove texture. The main interest is the possibility to obtain a cartoon image without texture.

\subsection{Image restoration}

A $512 \times 512$ pixels image was perturbed by an additive Gaussian noise of standard deviation $\sigma=0.1$. The resulting image is the House image, see Figure 7 (left). The results of the anisotropic restoration Algorithm 2 is presented in Figure 7 (center left). In order to illustrate the difference between Algorithm 1 (ID) and Algorithm 2 (AD), details are shown in Figure 8 (c) and (d). It can be seen that the restoration of edges is more accurate using the AD restoration.

A $512 \times 512$ pixels image was perturbed by an additive noise following a uniform distribution between $[-0.176,0.176]$. The resulting image is the Barbara image, see Figure 7 (center right). The results of the anisotropic 


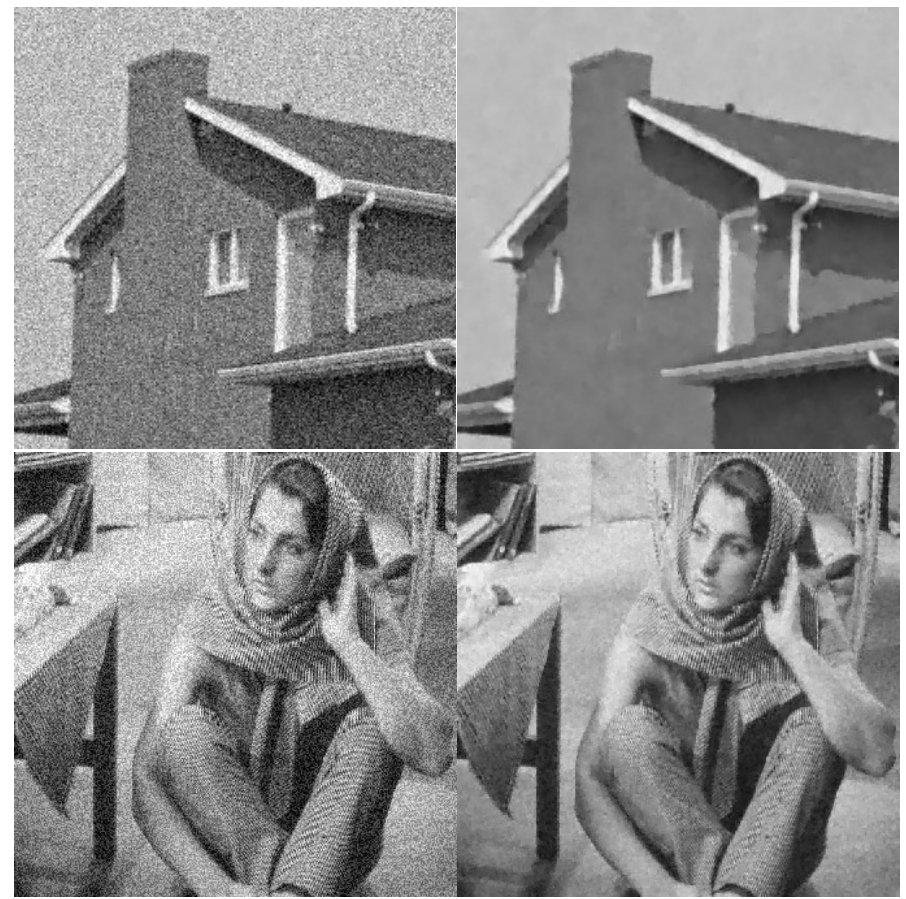

Figure 7. Noisy and AD restoration of House and Barbara images

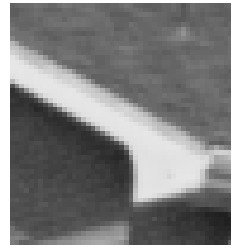

(a) Original image.

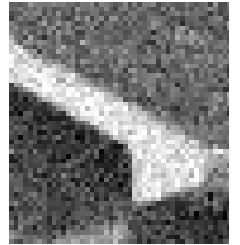

(b) Noisy image. (PSNR 20.94 dB)

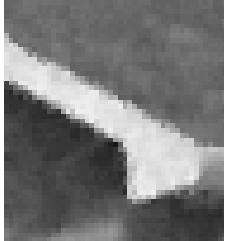

(c) ID restoration. (PSNR 29.47 dB)

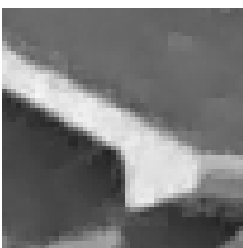

(d) AD restoration. (PSNR $31.46 \mathrm{~dB}$ )

Figure 8. Parts of House image (from left to right): original image, noisy image, ID and AD restorations.

restoration Algorithm 2 is presented in Figure 7 (right). The details presented in Figure 9 (c) and (d) show the interest of anisotropic diffusion along the edges.

In presence of an additive Gaussian noise, a comparative study with the Non-Local Means (NLM) 25] was performed to restore an image that is not composed of self-similar texture (Mandrill, Figure 10). We use the toolbox provided by [55]. 


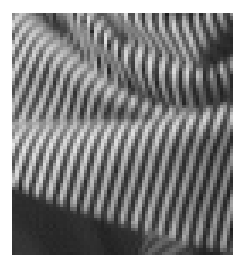

(a) Original image.

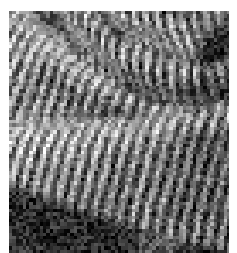

(b) Noisy image. (PSNR $19.86 \mathrm{~dB}$ )

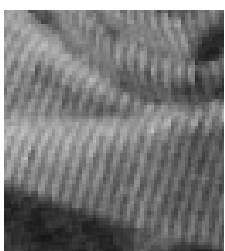

(c) ID restoration. (PSNR $25.09 \mathrm{~dB}$ )

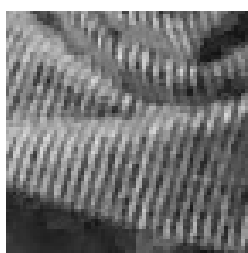

(d) AD restoration (PSNR 25.93 dB)

Figure 9. Parts of original Barbara image, noisy one, ID and $\mathrm{AD}$ restorations.

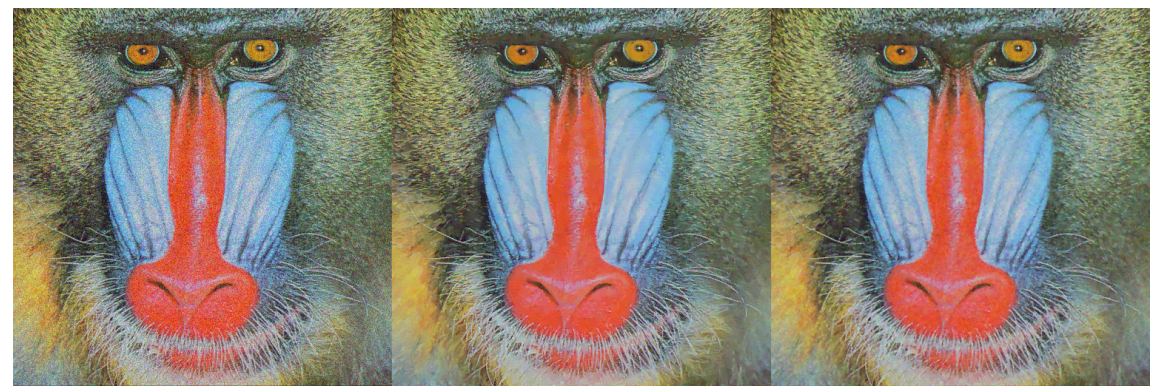

FIGURE 10. From right to left: noisy, NLM and AD restorations of Mandrill image.

It must be noted that on images that contain self-similar texture (e.g. Barbara), the results provided by the NLM outperform our diffusion method. This is sensible since in the NLM algorithm, the denoised value at a pixel $x$ is a mean of the values of all pixels whose Gaussian neighborhood looks like the neighborhood of $x$. The efficiency of this algorithm on a self similar image is therefore foreseeable.

\begin{tabular}{|c|c|c|c|}
\cline { 2 - 4 } \multicolumn{1}{c|}{} & \multicolumn{3}{c|}{ Mandrill Figure } \\
\cline { 2 - 4 } \multicolumn{1}{c|}{} & Noisy & NLM & AD \\
\hline PSNR & 20.16 & 22.63 & 23.00 \\
\hline SSIM & 0.552 & 0.708 & 0.702 \\
\hline
\end{tabular}

TABLE 2. Comparative results of restoration using NonLocal Means (NLM) and Algorithm 2 (AD).

Figure 10 shows the Mandrill $(512 \times 512 \times 3)$ color image with an additive Gaussian noise of standard deviation $\sigma=0.09$ and the restorations with NLM and AD. The three channels (RGB) were treated separately. The Non-Local 


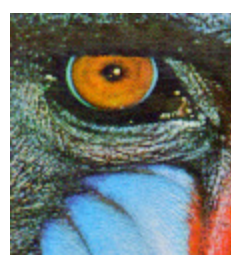

(a) Original image.

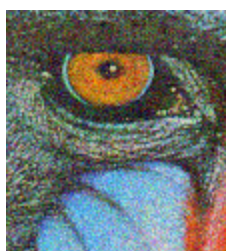

(b) Noisy image. (PSNR 20.16 dB)

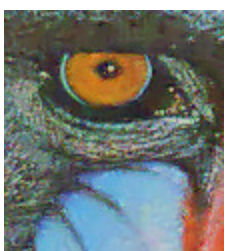

(c) Non-Local Means. (PSNR $22.63 \mathrm{~dB}$ )

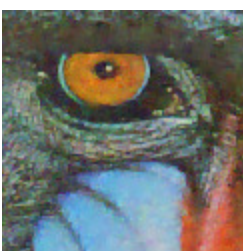

(d) AD restoration. (PSNR $23.00 \mathrm{~dB}$ )

Figure 11. Parts of original Mandrill image, noisy one, NLM and AD restorations.

Means and $\mathrm{AD}$ restorations provide similar results in terms of quality (see Table 2 and Figure 11 (c) and (d)).

This restoration algorithm is not only useful to suppress noise, it can also help removing JPEG artifacts. A $512 \times 512 \times 3$ pixels color image is perturbed by a JPEG compression using the GNU Image Manipulation Program [36]. The resulting image is the Peppers image, see Figure 12 (left). The results of the anisotropic restoration Algorithm 2 is presented in Figure 12 (right).
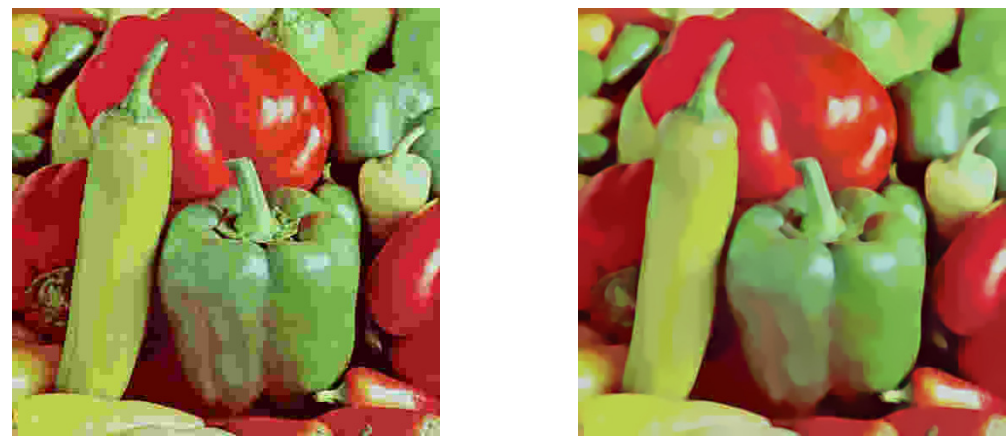

Figure 12. Suppression of JPEG artefacts.

\subsection{Image segmentation}

The objective of cartoon plus texture decomposition is to remove the texture of an image while preserving its main features. This can be achieved by using a large diffusion coefficient in Equation (6.2). In order to prevent edge degradation, the following isotropic diffusion coefficient was used:

$$
c(x)= \begin{cases}\epsilon & \text { in } \omega \\ \frac{1}{\epsilon} & \text { outside } \omega\end{cases}
$$


where $\omega \subset \Omega$ represent the edge set. The following problem adapted from (6.2) was solved:

$$
\left\{\begin{aligned}
-\nabla \cdot\left(\epsilon \nabla u_{\epsilon}\right)+u_{\epsilon} & =v \text { in } \omega \\
-\nabla \cdot\left(\frac{1}{\epsilon} \nabla u_{\epsilon}\right)+u_{\epsilon} & =v \text { in } \Omega \backslash \omega, \\
\partial_{n} u & =0 \text { on } \partial \Omega,
\end{aligned}\right.
$$

The segmentation provides an image composed of homogeneous regions. The suppression of texture or the separation of an image between cartoon part (smooth regions) and texture part is a developing task [12, 64. Figure 13 presents a texture removal example with an image of a cross stitch.
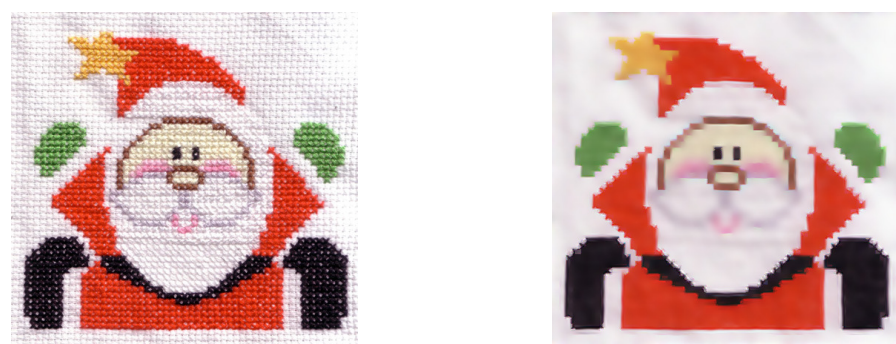

Figure 13. Texture removal.

\section{The observation operator $L$ is a mask operator}

Let $\omega$ be the missing part of the image. The observation operator is $L$ : $L^{2}(\Omega) \mapsto L^{2}(\Omega \backslash \omega)$ defined by $L u=\left.u\right|_{\Omega \backslash \omega}$. To solve inpainting problems, the following functional is minimized with $u \in H^{1}(\Omega)$ :

$$
\left\|c^{1 / 2} \nabla u\right\|_{L^{2}(\Omega)}^{2}+\|L u-v\|_{L^{2}(\Omega \backslash \omega)}^{2},
$$

where the anisotropic tensor $c(x)$ is computed using Algorithm 2

Section 7.1 addresses the problem of denoising a salt and pepper image, by considering that $\omega$ is the union of the pixels where the gray-level is saturated in black or white (gray-level 0 or 255 for 8-bit images). Section 7.2 proposes a unified approach to perform demosaicing and image denoising simultaneously.

\subsection{Salt and Pepper denoising}

In Figure 14, the gray-level Lena image $(1024 \times 1024)$ suffers from a Salt and Pepper noise, and $80 \%$ of the pixels are affected. The pixels in black and white are identified, their union is the unknown region $\omega$. The results of Algorithm 1 and Algorithm 2 on this problem are presented in Figure 14. The anisotropic diffusion provides better results in term of visual quality and PSNR assessment. 


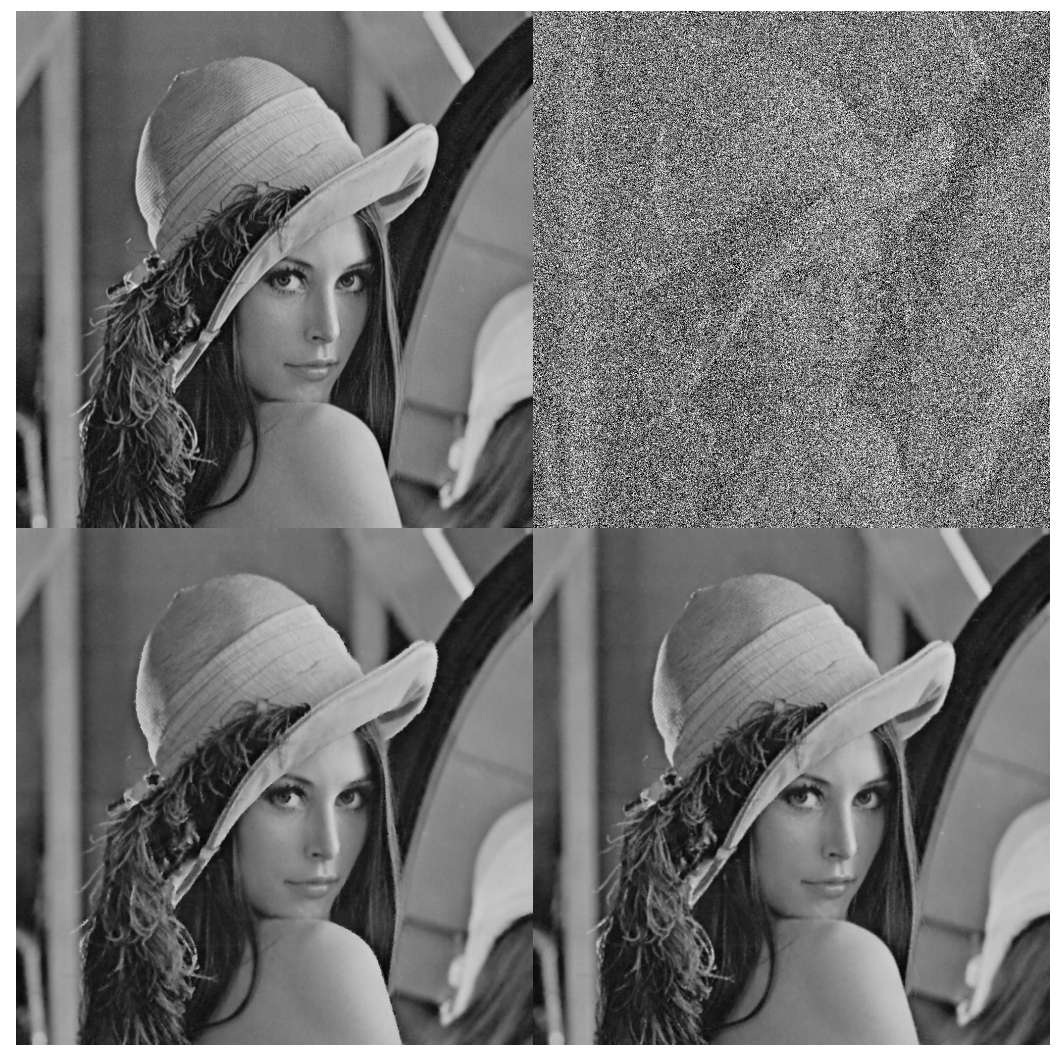

Figure 14. From left to right, first line, original Lena image and noisy image (PSNR $6.30 \mathrm{~dB}$ ), second line, isotropic restoration (PSNR $32.78 \mathrm{~dB}$ ) and anisotropic restoration (PSNR 35.66 dB).

\subsection{Demosaicing and denoising}

Demosaicing, also called the color filter array (CFA) interpolation, refers to the problem of reconstructing a color image from the charge-coupled device (CCD) samples. A color filtering array is a mosaic of color filters in front of the image sensor. While there are many kinds of color filters in use in digital cameras, the most commonly used is the Bayer filter. It is a grid composed of squares representing the three primary colors, see Figure 15 . To be noted that there are twice as many green photo sensors as red or blue, as the human eye is more sensitive to green light.

To reconstruct a color image from the uncompleted data, interpolation is needed to fill in the blanks. This specific inpainting application is called demosaicing. Demosaicing and image denoising are treated simultaneously with our method and the results are presented in Figure 16. 
24 Stanislas Larnier, Jérôme Fehrenbach and Mohamed Masmoudi

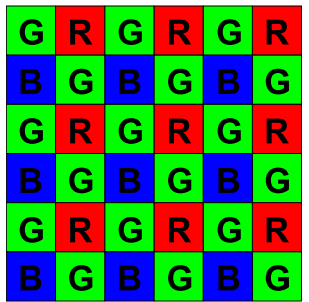

Figure 15. The Bayer filter grid.

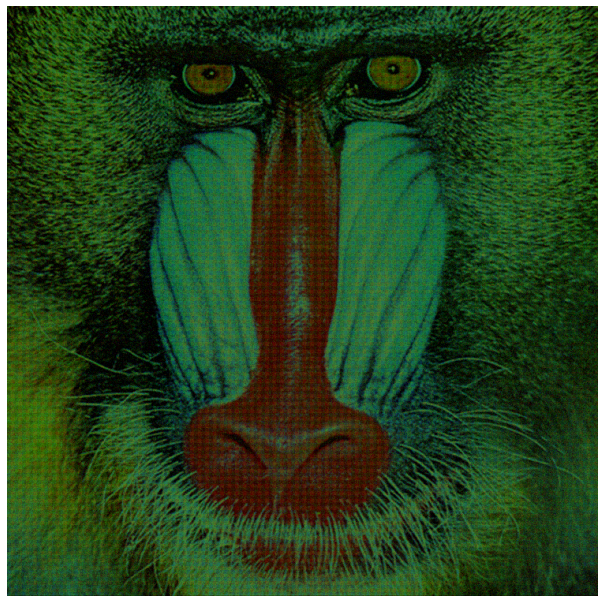

(a) Noisy Bayer filtered image.

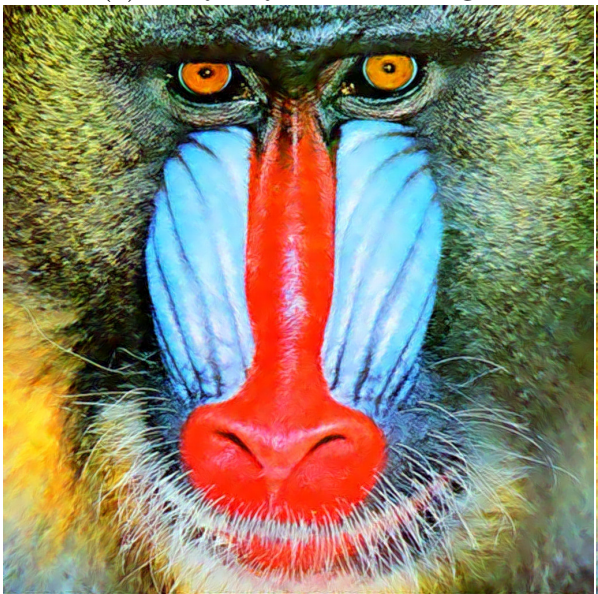

(c) TLS restoration.

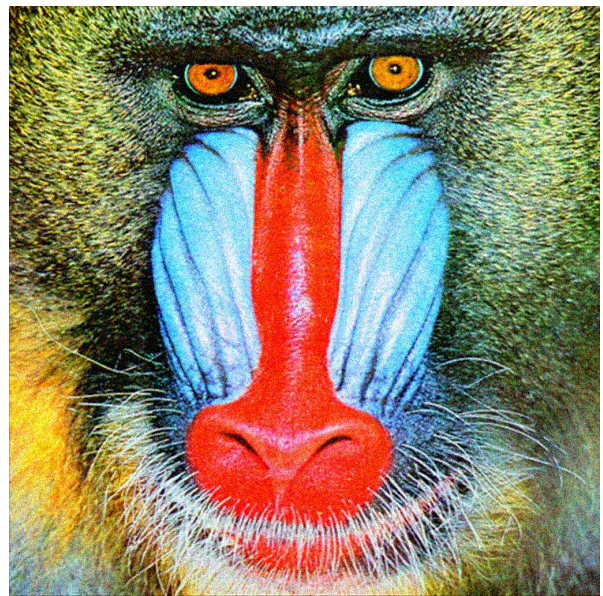

(b) AP restoration.

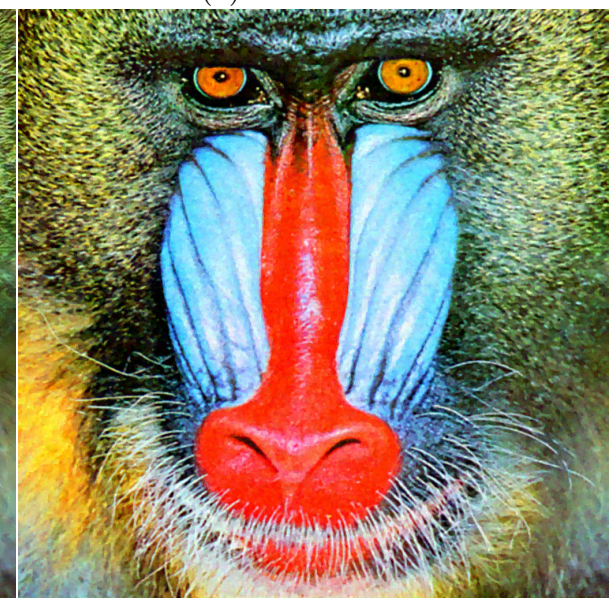

(d) AD restoration.

Figure 16. Demosaicing and denoising of a color image. 
For this example, the Mandrill original image $(1024 \times 1024 \times 3)$ is used. Figure 16 (a) is a Bayer filtered image perturbed by an additive Gaussian noise of standard deviation $\sigma=0.1$. Algorithm 2 was compared with two existing methods. Figure 16 (b) presents the reconstruction with the Alternating Projection technique (AP) 40. The AP is a benchmark method in demosaicing applications, and is not designed for denoising. The result is given to get an idea of the noise amount.

The coupling demosaicing-denoising has been treated in 41] using a Total Least Square method (TLS). The result of the TLS image denoising method from [4] is presented in Figure 16 (c). The reconstruction obtained with Algorithm 2 is Figure 16 (d). The quality of the results and computational aspects are summarized in Table 3. Is is clear that our Algorithm 2 provides similar results in terms of quality as Total Least Square and this with a lower computational load.

\begin{tabular}{|c|c|c|c|}
\hline & \multicolumn{3}{|c|}{ Mandrill Figure 16} \\
\hline & $\mathrm{AP}$ & TLS & $\overrightarrow{\mathrm{AD}}$ \\
\hline PSNR & 19.85 & 23.89 & 24.62 \\
\hline SSIM & 0.5119 & 0.7438 & 0.7600 \\
\hline $\mathrm{CPU}(\mathrm{s})$ & 14 & 3420 & 170 \\
\hline
\end{tabular}

TABLE 3. Comparative results for demosaicing and denoising using Alternative Projections (AP), Total Least Square (TLS) and Algorithm 2 (AD).

\section{Case of super-resolution}

The purpose of super-resolution techniques is to obtain a high resolution image from one or several low resolution images [52, 37]. Let $k$ be the number of low resolution images. The observation operator is $L: L^{2}(\Omega) \rightarrow E=$ $\left(\mathbb{R}^{m \times n}\right)^{k}$ and it is the composition of a convolution by a filter followed by $k$ subsampling operators. In the examples below a Gaussian noise is added to the observations.

To solve super-resolution problems, the following functional is minimized with $u \in H^{1}(\Omega)$ :

$$
\left\|c^{1 / 2} \nabla u\right\|_{L^{2}(\Omega)}^{2}+\|L u-v\|_{E}^{2},
$$

where the anisotropic tensor $c(x)$ is computed using Algorithm 2

Section 8.1 shows the capability of Algorithm 2 to treat one or many noisy filtered and sub-sampled images. In Section 8.2, a comparison with a Total Variation algorithm is proposed. 


\subsection{Super-resolution with one or many filtered and sub-sampled images}

For this example, the original Lena color image $(512 \times 512 \times 3)$ is used. The image is convolved with a Gaussian $5 \times 5$ filter with standard deviation $\tau=5$. The sub-sampling operation keeps one pixel out of nine and three lowresolution images are sub-sampled differently. An additive Gaussian noise of standard deviation $\sigma=0.075$ is added to each low-resolution image. In Figure 17 (center), only one filtered and sub-sampled image is used for the reconstruction. In Figure 17 (right), the observations are $k=3$ filtered and sub-sampled images.
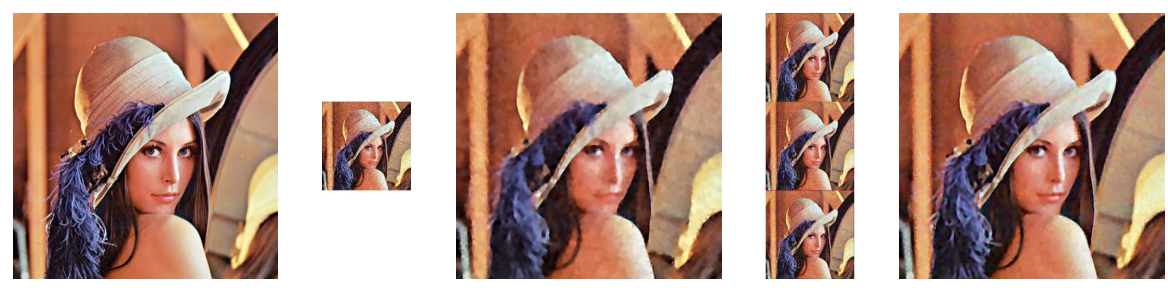

FiguRE 17. Images from left to right: original, noisy filtered and sub-sampled, restored (PSNR $19.28 \mathrm{~dB}$ ), three noisy filtered and sub-sampled, restored (PSNR 20.24 dB).

\subsection{Comparison with Total Variation algorithm}

The results of Algorithm 2 were compared with a TV- $L^{2}$ algorithm [45, 48. A grey-level image is sub-sampled by a factor 2 in each direction with a mean $2 \times 2$ filter. A Gaussian noise with standard deviation $\sigma=0.05$ is added to the sub-sampled image. Figure 18 presents the original Man image $(512 \times 512)$, the observed image, the image restored with a TV- $L^{2}$ algorithm and the image recovered using Algorithm 2. Table 4 presents quantitative estimators of the quality of reconstructions, the tests are performed on Man, Barbara and Boat images. The quality of the reconstruction with our Algorithm 2 outperforms the TV- $L^{2}$ method.

\begin{tabular}{|c|c|c|c|c|c|c|}
\cline { 2 - 7 } \multicolumn{1}{c|}{} & \multicolumn{2}{c|}{ Man } & \multicolumn{2}{c|}{ Barbara } & \multicolumn{2}{c|}{ Boat } \\
\cline { 2 - 7 } \multicolumn{1}{c|}{} & TV & AD & TV & AD & TV & AD \\
\hline PSNR & 24.81 & 28.07 & 22.63 & 24.32 & 24.03 & 27.41 \\
\hline SSIM & 0.652 & 0.753 & 0.598 & 0.623 & 0.626 & 0.728 \\
\hline
\end{tabular}

TABLE 4. Comparative results of super-resolution using TV$L^{2}$ optimization (TV) and Algorithm 2 (AD). 

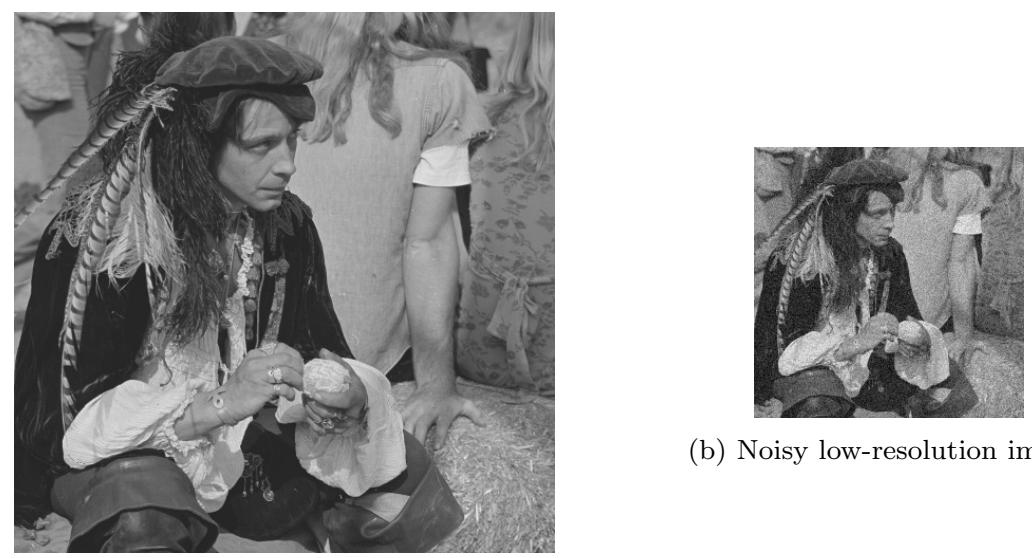

(b) Noisy low-resolution image.

(a) Original image.

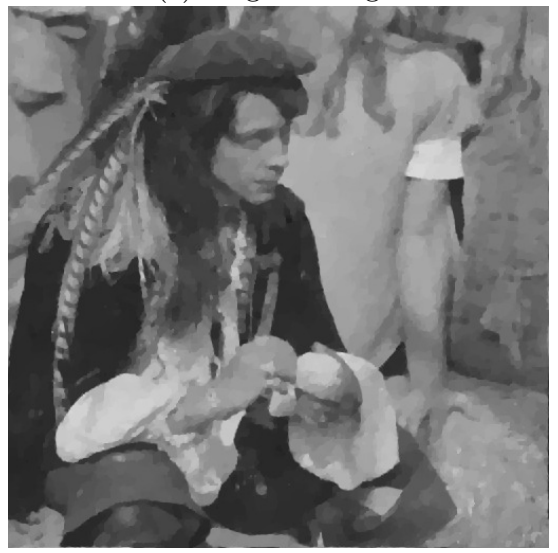

(c) TV restoration.

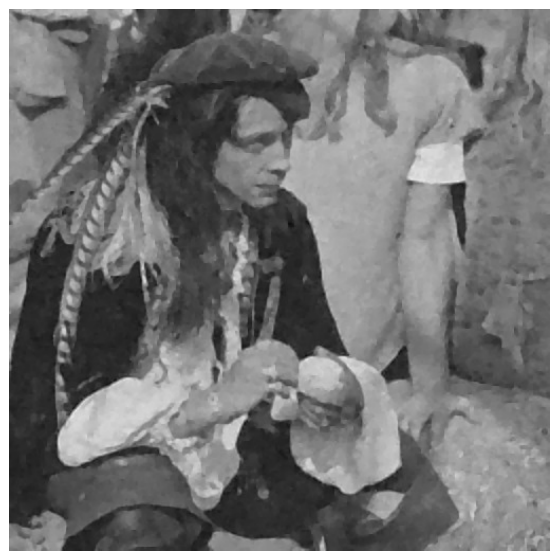

(d) AD restoration.

FiguRE 18. Super resolution of Man image.

\section{Conclusion}

This paper recalls that the crack detection technique based on the topological gradient can be applied to many applications in image processing. It provides an excellent frame for solving all these image processing problems. This technique can be applied to color images as well as grey-level images, but also three-dimensional images, or movies, without any trouble.

This work presents too a general approach to image processing using topological asymptotic analysis. This approach leads to the proposal of a general reconstruction algorithm (Algorithm 2) that incorporates two novelties: the observation operator $L$ is a general linear operator satisfying mild hypotheses (Hypothesis 4.1), and an anisotropic diffusion is performed to reconstruct the image. The fact that the observation operator is general allows an extension of the scope of topological asymptotic methods in image 
processing to new problems: image demosaicing and super-resolution image reconstruction. The introduction of anisotropic diffusion improve the results obtained with existing algorithms using topological gradient in image restoration and inpainting (Algorithm 1). The results obtained were also compared to reference methods, and show better or similar quality in terms of PSNR and SSIM.

\section{Acknowledgments}

The authors thanks particularly Pierre Weiss for providing his Total Variation codes and helpful discussions concerning this work. They also give thanks to Bahadir K. Gunturk, Keigo Hirakawa, Gabriel Peyre and Zhou Wang for their codes.

\section{References}

1. G. Alessandrini and A. Diaz Valenzuela, Unique determination of multiple cracks by two measurements, SIAM Journal on Control and Optimization 34 (1996), no. 3, 913-921.

2. G. Allaire, Shape optimization by the homogenization method, vol. 146, SpringerVerlag, Applied Mathematical Sciences, 2002.

3. G. Allaire, E. Bonnetier, G. Francfort, and F. Jouve, Shape optimization by the homogenization method, Numerische Mathematik 76 (1997), 27-68.

4. G. Allaire, F. de Gournay, F. Jouve, and A.-M. Toader, Structural optimization using topological and shape sensitivity via a level set method, Control and Cybernetics 34 (2005), 59-80.

5. G. Allaire and F. Jouve, A level-set method for vibration and multiple loads structural optimization, Computer Methods in Applied Mechanics and Engineering 194 (2005), no. 30-33, 3269 - 3290, Structural and Design Optimization.

6. S. Amstutz, The topological asymptotic for the navier-stokes equations, ESAIM: Control, Optimisation and Calculus of Variations 11 (2005), 401-425.

7. S. Amstutz, I. Horchani, and M. Masmoudi, Crack detection by the topological gradient method, Control and Cybernetics 34 (2005), no. 1, 81-101.

8. S. Amstutz, M. Masmoudi, and B. Samet, The topological asymptotic for the helmoltz equation, SIAM Journal on Control Optimization 42 (2003), no. 5, 1523-1544.

9. S. Amstutz, T. Takahashi, and B. Vexler, Topological sensitivity analysis for time-dependent problems, ESAIM: Control, Optimisation and Calculus of Variations 14 (2008), 427-455.

10. S. Andrieux and A. Ben Abda, Identification of planar cracks by complete overdetermined data: inversion formulae, Inverse Problems 12 (1996), 553-563.

11. G. Aubert and P. Kornprobst, Mathematical problems in image processing: Partial differential equations and the calculus of variations, vol. 147, SpringerVerlag, Applied Mathematical Sciences, November 2001. 
12. J.-F. Aujol, G. Gilboa, T. Chan, and S. Osher, Structure-texture image decomposition - modeling, algorithms, and parameter selection, International Journal of Computer Vision 67 (2006), no. 1, 111-136.

13. D. Auroux, L. Jaafar Belaid, and B. Rjaibi, Application of the topological gradient method to color image restoration, SIAM J. Imaging Sci. 3 (2010), no. 2, 153-175.

14. D. Auroux and M. Masmoudi, A one-shot inpainting algorithm based on the topological asymptotic analysis, Computational and Applied Mathematics 25 (2006), no. 2-3, 251-267.

15. D. Auroux and M. Masmoudi, Image processing by topological asymptotic analysis, ESAIM: Proc. Mathematical methods for imaging and inverse problems 26 (2009), 24-44.

16. D. Auroux and M. Masmoudi, Image processing by topological asymptotic expansion, Journal of Mathematical Imaging and Vision 33 (2009), no. 2, 122-134.

17. L. J. Belaid, M. Jaoua, M. Masmoudi, and L. Siala, Image restoration and edge detection by topological asymptotic expansion, C. R. Acad. Sci. Paris 342 (2006), no. 5, 313-318.

18. Z. Belhachmi and D. Bucur, Stability and uniqueness for the crack identification problem, SIAM Journal on Control and Optimization 46 (2007), no. 1, 253-273.

19. A. Ben Abda, H. Ben Ameur, and M. Jaoua, Identification of $2 d$ cracks by elastic boundary measurements, Inverse Problems 15 (1999), 67-77.

20. A. Ben Abda, M. Kallel, J. Leblond, and J.-P. Marmorat, Line-segment cracks recovery from incomplete boundary data, Inverse Problems 18 (2002), 10571077.

21. M. Bendsoe and O. Sigmund, Topology optimization. theory, methods and applications, Springer, 2003.

22. M. P. Bendse and N. Kikuchi, Generating optimal topologies in structural design using a homogenization method, Computer Methods in Applied Mechanics and Engineering 71 (1988), no. 2, $197-224$.

23. M. Bruhl, M. Hanke, and M. Pidcock, Crack detection using electrostatic measurements, Mathematical Modelling and Numerical Analysis 35 (2001), 595605.

24. K. Bryan and M.S. Vogelius, A review of selected works on crack identification, Proceedings of the IMA workshop on Geometric Methods in Inverse Problems and PDE Control, August 2001.

25. A. Buades, B. Coll, and J.M Morel, A review of image denoising algorithms, with a new one, Multiscale Modeling and Simulation (SIAM interdisciplinary journal) 4 (2005), no. 2, 490-530.

26. A. P. Calderón, On an inverse boundary value problem, Seminar on Numerical Analysis and Its Applications to Continuum Physics (Rio de Janeiro, 1980) (Rio de Janeiro, Brasil), Soc. Brasil. Mat., 1980, pp. 65-73.

27. F. Catté, P.-L. Lions, J.-M. Morel, and T. Coll, Image selective smoothing and edge detection by nonlinear diffusion, SIAM Journal on Numerical Analysis 29 (1992), no. 1, 182-193.

28. T. Chan and J. Shen, Mathematical models for local deterministic inpaintings, UCLA CAM Tech. Report 00-11 (2000). 
29. Non-texture inpainting by curvature-driven diffusions (ccd), UCLA CAM Tech. Report 00-35 (2000).

30. Image processing and analysis: variational, pde, wavelet, and stochastic methods, SIAM, 2005.

31. M. Elad and M. Aharon, Image denoising via sparse and redundant representations over learned dictionaries, Image Processing, IEEE Transactions on $\mathbf{1 5}$ (2006), no. 12, $3736-3745$.

32. M Elad, J Starck, P Querre, and D Donoho, Simultaneous cartoon and texture image inpainting using morphological component analysis (mca), Applied and Computational Harmonic Analysis 19 (2005), no. 3, 340-358.

33. A. Friedman and M.S. Vogelius, Determining cracks by boundary measurements, Indiana University Mathematics Journal 38 (1989), no. 3, 527-556.

34. _ Identification of small inhomogeneities of extreme conductivity by boundary measurements: a theorem of continuous dependance, Archive for Rational Mechanics and Analysis 105 (1989), no. 4, 299-326.

35. S. Garreau, P. Guillaume, and M. Masmoudi, The topological asymptotic for pde systems: The elasticity case, SIAM Journal on Control and Optimization 39 (2001), no. 6, 1756-1778.

36. Gimp, The gnu image manipulation program, http://www.gimp.org.

37. D. Glasner, S. Bagon, and M. Irani, Super-resolution from a single image, Proceedings of the IEEE International Conference on Computer Vision (Kyoto, Japan), September 2009.

38. P. Guillaume and K. Sid Idris, The topological asymptotic expansion for the Dirichlet problem, SIAM Journal on Control and Optimization 41 (2002), no. 4, 1042-1072.

39. - The topological sensitivity and shape optimization for the Stokes equations, SIAM Journal on Control and Optimization 43 (2004), no. 1, 1-31.

40. B. K. Gunturk, Y. Altunbasak, and R. M. Mersereau, Color plane interpolation using alternating projections, Image Processing, IEEE Transactions on 11 (2002), $997-1013$.

41. K. Hirakawa and T.W. Parks, Joint demosaicing and denoising, Image Processing, IEEE Transactions on Image Processing 15 (2006), 2146-2157.

42. R. Kohn and M. Vogelius, Relaxation of a variational method for impedance computed tomography, Communications on Pure and Applied Mathematics 40 (1987), 745-777.

43. S. Kubo and K. Ohji, Inverse problems and the electric potential computed tomography method as one of their application, Mechanical Modeling of New Electromagnetic Materials (1990).

44. S. Larnier and J. Fehrenbach, Edge detection and image restoration with anisotropic topological gradient, Acoustics Speech and Signal Processing (ICASSP), 2010 IEEE International Conference on, march 2010, pp. 1362 1365 .

45. F. Malgouyres and F. Guichard, Edge direction preserving image zooming: a mathematical and numerical analysis, SIAM Journal on Numerical Analysis 39 (2002), no. 1, 1-37. 
46. M. Masmoudi, The topological asymptotic, Glowinski, R., Karawada, H., Periaux, J. (eds.) Computational Methods for Control Applications, GAKUTO international series, Mathematical science and applications 16 (2001), 53-72.

47. B. Mohammadi and O. Pironneau, Shape optimization in fluid mechanics, Annual Review of Fluid Mechanics 36 (2003), 255-279.

48. M. Ng, P. Weiss, and X.-M. Yuan, Solving constrained total-variation image restoration and reconstruction problems via alternating direction methods, SIAM journal on Scientific Computing (2010), Accepted for publication.

49. N. Nishimura and S. Kobayashi, A boundary integral equation method for an inverse problem related to crack detection, International Journal for Numerical Methods in Engineering 32 (1991), no. 7, 1371-1387.

50. S. Osher and F. Santosa, Level set methods for optimization problems involving geometry and constraints: I. frequencies of a two-density inhomogeneous drum, Journal of Computational Physics 171 (2001), no. 1, 272 - 288.

51. S. Osher and J. A Sethian, Fronts propagating with curvature-dependent speed: Algorithms based on Hamilton-Jacobi formulations, Journal of Computational Physics 79 (1988), no. 1, $12-49$.

52. S. C. Park, M. K. Park, and M. G. Kang, Super-resolution image reconstruction: a technical overview, Signal Processing Magazine, IEEE 20 (2003), no. 3, 21-36.

53. P. Perona and J. Malik, Scale-space and edge detection using anisotropic diffusion, Proc. IEEE Comp. Soc. Workshop on Computer Vision (Miami Beach, Nov. 30 Dec. 2, 1987) (1987), 1622.

54. Scale-space and edge detection using anisotropic diffusion, IEEE Trans. Pattern Anal. Mach. Intell. 12 (1990), no. 7, 629-639.

55. G. Peyre, Non-local means toolbox.

56. F. Santosa and M. Vogelius, A computational algorithm to determine cracks from electrostatic boundary measurements, International Journal of Engineering Science 29 (1991), no. 8, 917 - 937.

57. A. Schumacher, Topologieoptimisierung von bauteilstrukturen unter verwendung von lopchpositionierungkrieterien, Ph.D. thesis, Universitat-Gesamthochschule Siegen, Germany, 1995.

58. J. Sokolowski and A. Zochowski, On the topological derivative in shape optimization, SIAM Journal on Control and Optimization 37 (1999), 1241-1272.

59. D. Terzopoulos, Regularization of inverse visual problems involving discontinuities, Pattern Analysis and Machine Intelligence, IEEE Transactions on PAMI-8 (1986), no. 4, $413-424$.

60. M. Y. Wang, X. Wang, and D. Guo, A level set method for structural topology optimization, Computer Methods in Applied Mechanics and Engineering 192 (2003), no. 1-2, $227-246$.

61. Z. Wang, A. C. Bovik, H. R. Sheikh, and E. P. Simoncelli, Image quality assessment : From error visibility to structural similarity, IEEE Transactions on Image Processing 13 (2004), no. 4, 600-612.

62. J. Weickert, Anisotropic diffusion in image processing, Ph.d. thesis, Dept. of Mathematics, University of Kaiserslautern, Germany, 1996.

63. _ Theoretical foundations of anisotropic diffusion in image processing, Computing, Suppl 11 (1996), 221-236. 
64. P. Weiss, L. Blanc-Fraud, and G. Aubert, Efficient schemes for total variation minimization under constraints in image processing, SIAM journal on Scientific Computing 31 (2009), no. 3, 2047-2080.

65. P. Wen, X. Wu, and C. Wu, An interactive image inpainting method based on rbf networks, Advances in Neural Networks - ISNN 2006, Lecture Notes in Computer Science, vol. 3972, Springer Berlin / Heidelberg, 2006, pp. 629-637.

66. T. Zhou, F. Tang, J. Wang, Z. Wang, and Q. Peng, Digital image inpainting with radial basis functions, Journal of Image and Graphics 9 (2004), no. 10, 1190-1196.

\section{Stanislas Larnier}

Institut de Mathématiques de Toulouse,

Université Paul Sabatier, 118 route de Narbonne,

F-31062 Toulouse Cedex 9, France

e-mail: stanislas.larnier@math.univ-toulouse.fr

Jérôme Fehrenbach

Institut de Mathématiques de Toulouse,

Université Paul Sabatier, 118 route de Narbonne,

F-31062 Toulouse Cedex 9, France

e-mail: jerome.fehrenbach@math.univ-toulouse.fr

Mohamed Masmoudi

Institut de Mathématiques de Toulouse,

Université Paul Sabatier, 118 route de Narbonne,

F-31062 Toulouse Cedex 9, France

e-mail: mohamed.masmoudi@math.univ-toulouse.fr 